\title{
Survival outcome and mortality rate in patients with migraine: a population-based cohort study
}

\author{
Tomor Harnod ${ }^{1,2}$, Cheng-Li Lin ${ }^{3,4}$ and Chia-Hung Kao 5,6,7*
}

\begin{abstract}
Background: Whether the patients with migraine have an elevated mortality risk in Taiwan is unclear.

Methods: We analyzed a subset of the National Health Insurance Research Database of Taiwan and enrolled patients ( $\geq 20$ years old) who received a diagnosis of migraine between 2000 and 2012. The migraine cohort was further divided into the ones ever with status migrainosus (SM) and non-status migraine (NM) subcohort and compared with a 1:4 age-, sex-, comorbidity-, and index date-matched comparison cohort. We calculated the adjusted hazard ratios (aHRs) and 95\% confidence intervals (Cls) for subsequent mortality risk after adjustment for age, sex, and comorbidities.

Results: Compared with the comparison cohort, the corresponding aHRs for mortality were $0.81(95 \% \mathrm{Cl}=0.76-0.87)$, $0.89(95 \% \mathrm{Cl}=0.80-0.98)$, and $0.78(95 \% \mathrm{Cl}=0.72-0.84)$ in the total migraine, SM, and NM cohorts, respectively. SM, male sex, comorbid alcohol-related illness, depression, and mental disorders were identified as risk factors for subsequent mortality. Comorbid alcohol-related illness significantly increased the mortality risk in patients with migraine.

Conclusion: Taiwanese patients with migraine require comprehensive and universal medical care. These patients would benefit from controlling their migraines and reducing the subsequent mortality.
\end{abstract}

Keywords: Cohort study, Migraine, Mortality, National Health Insurance

\section{Background}

Migraine is a major disabling disease worldwide. Numerous epidemiological studies conducted in developed Western countries have found that migraines affect approximately $20 \%$ of the general population $[1,2]$. An international study in developing Asian countries showed that migraine accounts for $66.6 \%$ (range: $50.9 \%-85.8 \%$ ) of all headache services at neurological clinics [3]. Globally, migraine is approximately 2 times more prevalent in women than in men, particularly in young and middle-aged women during menstruation $[2,4,5]$. Migraine not only affects the central nervous system and disrupts daily life but also increases life-threatening comorbid psychological and cardiovascular diseases in

\footnotetext{
* Correspondence: d10040@mail.cmuh.org.tw; dr.kaochiahung@gmail.com ${ }^{5}$ Graduate Institute of Biomedical Sciences and School of Medicine, College of Medicine, China Medical University, No. 2, Yuh-Der Road, Taichung 404 Taiwan

${ }^{6}$ Department of Nuclear Medicine and PET Center, China Medical University Hospital, Taichung, Taiwan
}

Full list of author information is available at the end of the article patients $[6,7]$. The prevalence for migraine has been investigated a lot for the past 50 years and described the burden of migraine on individuals and communities worldwide $[5,8]$. Researchers have proposed that patients with migraine have a higher risk of mortality due to a high vulnerability to other fatal diseases. Although numerous studies have examined the markers for the long-term outcomes of migraine, the actual mortality risk in patients with migraine has remained unclear so far $[5,8,9]$. Therefore, further investigation is required to understand the correlated mortality in patients with migraine to elucidate future treatment strategies for migraine.

Treatment-resistant migraine or status migrainosus (SM) poses a unique and difficult challenge to headache specialists. Patients with such a condition may experience considerably more disability than patients with a classic, non-status migraine (NM) and thus may require specific pharmacological treatment or adopt psychological strategies to reduce the burden of the disease [10]. There might be different mortality risks in these 2 
groups of patients compared with the general population. We used a nationwide, population-based database to investigate the subsequent mortality rates in patients ever with SM and NM. The findings of this study might constructively inform the development and implementation of effective treatment strategies to reduce the migraine burden in Taiwan.

\section{Methods}

\section{Data source}

This population-based cohort study used the Longitudinal Health Insurance Database (LHID) provided by the National Health Research Institutes in Taiwan. Since March 1995, the Taiwanese government has managed a National Health Insurance (NHI) program that provides comprehensive universal health insurance to approximately $99.9 \%$ of the Taiwan population (more than 23 million individuals) $[11,12]$. The details of the LHID and NHI program have been well documented [13, 14]. In this study, diagnoses were coded according to the International Classification of Diseases, Ninth Revision, Clinical Modification (ICD-9-CM). This study was approved by the Ethics Review Board of China Medical University in Taiwan (CMUH-104-REC2-115).

\section{Participants}

The migraine cohort was composed of patients with newly diagnosed SM (ICD-9-CM code 346.9, and without code 346.90 or 346.91 ) or NM (ICD-9-CM code 346.x except 346.9) from January 1, 2000 to December 31, 2012. The date of the diagnosis was defined as the index date. The comparison cohort comprised patients without a migraine diagnosis in the LHID. For each identified patient with migraine, 4 non-migraine participants from the LHID were randomly selected for the comparison cohort and were frequency matched with age (each 5-year span); sex; and comorbidities of depression (ICD-9-CM 296.2, 296.3, 296.82, 300.4, and 311), mental disorders (ICD-9-CM 290-319), insomnia (ICD-9-CM 307.4 and 780.5), alcohol-related illness (ICD-9-CM 291, 303, 305.00, 305.01, 305.02, 305.03, 571.0, 571.1, 571.3, 790.3, and V11.3), and anxiety (ICD-9-CM 300.00); and index date (year). Patients aged $<20$ years or with incomplete demographic information were excluded (Fig. 1). The participants were followed until death, withdrawal from the NHI, or the end of 2013, whichever occurred first.

\section{Statistical analysis}

The distributions of demographic characteristics, including age, sex, and comorbidities, were compared between the migraine and non-migraine cohorts. The differences were examined using the chi-square and $t$ tests for categorical and continuous variables, respectively. We calculated the incidence density rates of subsequent mortality for the total migraine, SM, NM, and comparison cohorts. Univariable and multivariable Cox proportional hazard regression analyses were used to assess the risk of mortality associated with migraine compared with the comparison cohort. Hazard ratios (HRs) and 95\% confidence intervals (CIs) were estimated in the Cox models. In a multivariable model, we adjusted for age, sex, and comorbidities of depression, mental disorders, insomnia, alcohol-related illness, and anxiety, all of

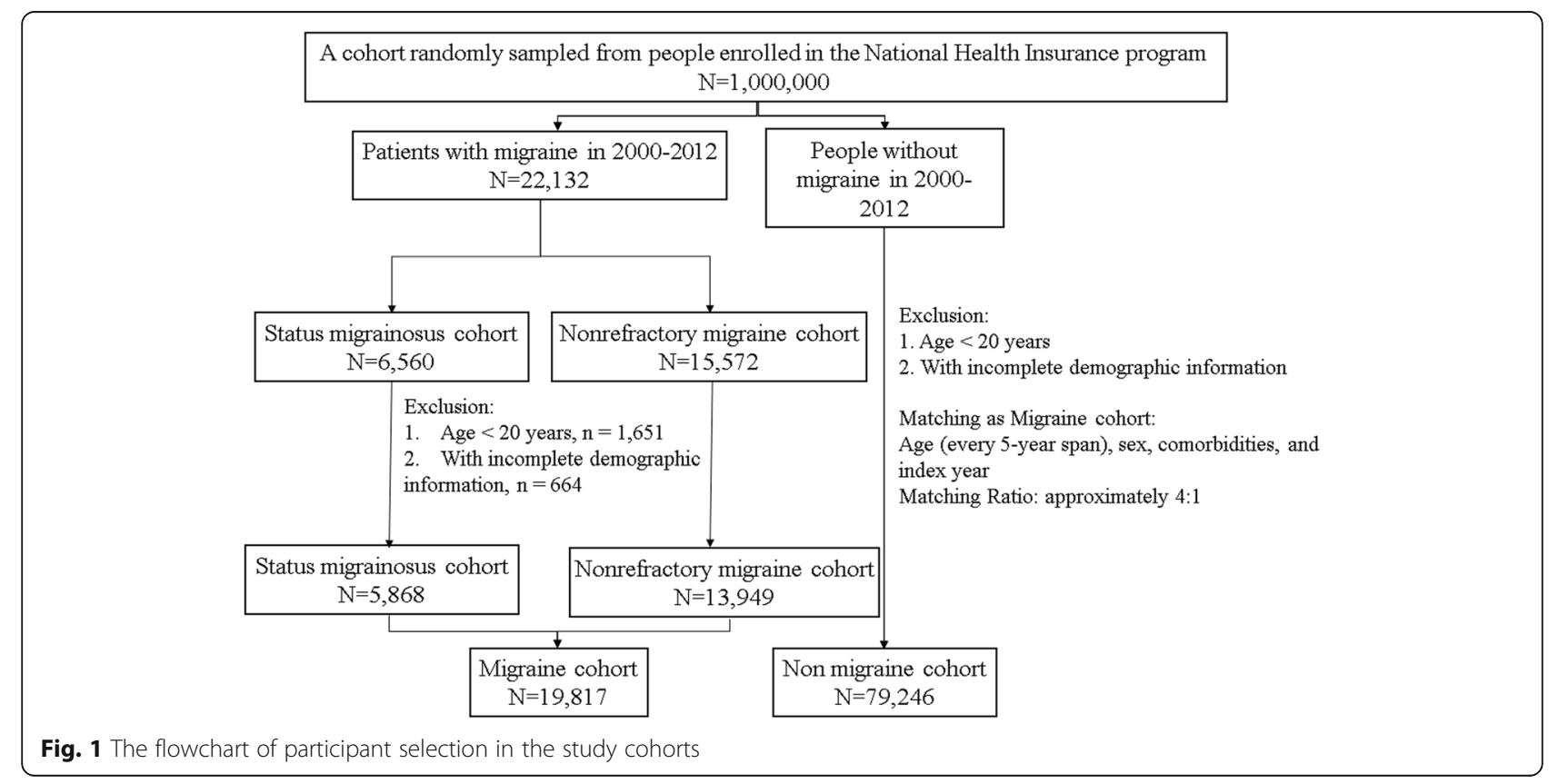


which had a significant difference in the univariable model. A multivariable regression analysis was used to estimate the average number of hospital days per year for all-cause admissions and frequency of migraine-related medical visits annually, and those associated with the risk factors in patients with migraine were determined using a stepwise selection method. We used SAS (version 9.4, Statistical Analysis System Institute Inc., Cary, NC, USA) for the data analyses. A two-tailed $P$ value of $<0.05$ was considered indicative of statistical significance.

\section{Results}

In total, 19,817 patients with migraine were selected (70.4\% NM and $29.6 \%$ ever with SM). In both migraine and comparison cohorts, the mean age was 46 years (standard deviation $[\mathrm{SD}]=15$ ), proportion of women was $73.3 \%$, and insomnia was the major comorbidity, followed by mental disorders and anxiety (Table 1).

The overall incidence density rates of mortality were 9.74, 7.95, 9.02, and 7.49 per 1000 person-years in the comparison, total migraine, SM, and NM cohorts, respectively (Table 2). Compared with the comparison cohort, the corresponding adjusted HRs (aHRs; 95\% CIs) of the mortality were 0.81 (0.76-0.87), 0.89 (0.80-0.98), and $0.78(0.72-0.84)$ for the total migraine, SM, and NM cohorts, respectively. Patients aged $50-64$ years $(\mathrm{aHR}=2.57$, $95 \% \mathrm{CI}=2.40-2.75)$ and $\geq 65$ years $(\mathrm{aHR}=10.5,95 \%$ $\mathrm{CI}=9.84-11.1)$ had a higher risk of mortality when compared with those aged $20-49$ years. In the multivariable model, the mortality risk was higher in men $(\mathrm{aHR}=1.78$, 95\% $\mathrm{CI}=1.70-1.87)$ and in patients with comorbid depression $(\mathrm{aHR}=1.33,95 \% \mathrm{CI}=1.24-1.43)$, mental disorders $(\mathrm{aHR}=1.19,95 \% \mathrm{CI}=1.12-1.27)$, and alcohol-related illness $(\mathrm{aHR}=2.18,95 \% \mathrm{CI}=1.98-2.40)$. Furthermore, the NM cohort had a significantly lower mortality risk $(\mathrm{aHR}=0.89,95 \% \mathrm{CI}=0.79-0.99)$ than did the SM cohort (Table 2).

Table 3 shows that the mortality risk, stratified by sex, age, and comorbidities; it was significantly lower in the total migraine and NM cohorts than in the comparison cohort. The aHRs of mortality were $0.68-0.86$ and $0.64-$ 0.84 in the total migraine and NM cohorts, respectively. In the total migraine cohort, patients with comorbidities demonstrated higher mortality rate than did those without. In patients with $\mathrm{SM}$, the relative risk of mortality was lower in those aged 20-49 years $(\mathrm{aHR}=0.79,95 \%$ $\mathrm{CI}=0.64-0.98)$ and in those without comorbidities $(\mathrm{aHR}=0.73,95 \% \mathrm{CI}=0.57-0.94$; Table 3$)$.

Table 4 shows the stepwise regression analysis for evaluating the factors associated with the average all-cause hospital days annually among the patients with migraine. Compared with the comparison cohort, the average hospitalization duration was 0.66 days longer for the patients ever with SM. Furthermore, alcohol-related illness, male sex, depression, mental disorders, and age increased the hospitalization duration by $4.10,1.98,1.08$, 0.81 , and 0.11 days in the patients with migraine (Table 4). Table 5 shows the stepwise regression analysis used to evaluate the factors associated with the frequency of

Table 1 Distribution of age, sex, and comorbidities between the migraine and comparison cohorts

\begin{tabular}{|c|c|c|c|c|c|c|c|c|c|}
\hline & \multicolumn{6}{|c|}{ Migraine } & \multirow{2}{*}{\multicolumn{2}{|c|}{$\frac{\text { Comparison }}{N=79,246}$}} & \multirow[t]{3}{*}{$p$-value } \\
\hline & \multicolumn{2}{|c|}{$\begin{array}{l}\text { Total } \\
N=19,817\end{array}$} & \multicolumn{2}{|c|}{$\begin{array}{l}\text { Status migrainosus } \\
N=5868\end{array}$} & \multicolumn{2}{|c|}{$\begin{array}{l}\text { Non-status migraine } \\
N=13,949\end{array}$} & & & \\
\hline & $\bar{n}$ & $\%$ & $n$ & $\%$ & $\bar{n}$ & $\%$ & $n$ & $\%$ & \\
\hline Age, year & & & & & & & & & 0.99 \\
\hline $20-49$ & 12,641 & 63.8 & 3685 & 62.8 & 8956 & 64.2 & 50,552 & 63.8 & \\
\hline $50-64$ & 4683 & 23.6 & 1388 & 23.7 & 3295 & 23.6 & 18,732 & 23.6 & \\
\hline $65+$ & 2493 & 12.6 & 795 & 13.6 & 1698 & 12.2 & 9962 & 12.6 & \\
\hline Mean $(S D)^{a}$ & 45.9 & 14.9 & 46.3 & 15.3 & 45.7 & 14.8 & 45.8 & 15.2 & 0.37 \\
\hline Sex & & & & & & & & & 0.99 \\
\hline Female & 14,533 & 73.3 & 4199 & 71.6 & 10,334 & 74.1 & 5819 & 73.3 & \\
\hline Male & 5284 & 26.7 & 1669 & 28.4 & 3615 & 25.9 & 21,127 & 26.7 & \\
\hline \multicolumn{10}{|l|}{ Comorbidity } \\
\hline Depression & 2536 & 12.8 & 663 & 11.3 & 1873 & 13.4 & 10,129 & 12.8 & 0.95 \\
\hline Mental disorders & 10,928 & 55.1 & 3033 & 51.7 & 7895 & 56.6 & 43,690 & 55.1 & 0.97 \\
\hline Insomnia & 13,414 & 67.7 & 3774 & 64.3 & 9640 & 69.1 & 53,645 & 67.7 & 0.99 \\
\hline Alcohol-related illness & 746 & 3.76 & 199 & 3.39 & 547 & 3.92 & 2964 & 3.74 & 0.87 \\
\hline Anxiety & 7316 & 36.9 & 2045 & 34.9 & 5271 & 37.8 & 29,248 & 36.9 & 0.98 \\
\hline
\end{tabular}

Chi-square test; ${ }^{a} t$ test

${ }^{\mathrm{b}}$ Total migraine vs. comparison 
Table 2 Incidence and hazard ratios of mortality and associated risk factors

\begin{tabular}{|c|c|c|c|c|c|c|}
\hline & \multicolumn{6}{|c|}{ HR (95\% Cl) } \\
\hline & Event no & Person-years & Rate & Crude & Adjusted $^{a}$ & Adjusted $^{a}$ \\
\hline \multicolumn{7}{|l|}{ Migraine } \\
\hline None & 5567 & 582,126 & 9.74 & 1.00 & 1.00 & \\
\hline Total & 1167 & 146,776 & 7.95 & $0.82(0.77,0.87)^{* * *}$ & $0.81(0.76,0.87)^{* * *}$ & \\
\hline Status migrainosus & 401 & 44,471 & 9.02 & $0.92(0.83,1.02)$ & $0.89(0.80,0.98)^{*}$ & 1.00 \\
\hline Non-status migraine & 766 & 102,304 & 7.49 & $0.77(0.72,0.83)^{* * *}$ & $0.78(0.72,0.84)^{* * *}$ & $0.89(0.79,0.99)^{*}$ \\
\hline \multicolumn{7}{|l|}{ Age, year } \\
\hline $20-49$ & 1753 & 475,258 & 3.69 & 1.00 & 1.00 & 1.00 \\
\hline $50-64$ & 1667 & 171,118 & 9.74 & $2.65(2.48,2.84)^{* * * *}$ & $2.57(2.40,2.75)^{* * *}$ & $3.05(2.58,3.61)^{* * *}$ \\
\hline $65+$ & 3414 & 82,525 & 41.4 & $11.4(10.8,12.1)^{* * *}$ & $10.5(9.84,11.1)^{* * *}$ & $12.4(10.6,14.4)^{* * *}$ \\
\hline \multicolumn{7}{|l|}{ Sex } \\
\hline Female & 3693 & 538,264 & 6.86 & 1.00 & 1.00 & 1.00 \\
\hline Male & 3141 & 190,637 & 16.5 & $2.41(2.29,2.52)^{* * *}$ & $1.78(1.70,1.87)^{* * *}$ & $1.96(1.74,2.21)^{* * *}$ \\
\hline \multicolumn{7}{|l|}{ Comorbidity } \\
\hline \multicolumn{7}{|l|}{ Depression } \\
\hline No & 5771 & 643,710 & 8.97 & 1.00 & 1.00 & 1.00 \\
\hline Yes & 1063 & 85,192 & 12.5 & $1.41(1.32,1.50)^{* * *}$ & $1.33(1.24,1.43)^{* * *}$ & $1.27(1.07,1.52)^{* *}$ \\
\hline \multicolumn{7}{|l|}{ Mental disorders } \\
\hline No & 2279 & 320,327 & 7.11 & 1.00 & 1.00 & 1.00 \\
\hline Yes & 4555 & 408,575 & 11.2 & $1.56(1.49,1.64)^{* * *}$ & $1.19(1.12,1.27)^{* * *}$ & $1.19(1.02,1.39)^{*}$ \\
\hline \multicolumn{7}{|l|}{ Insomnia } \\
\hline No & 1985 & 263,064 & 7.55 & 1.00 & 1.00 & 1.00 \\
\hline Yes & 4849 & 465,838 & 10.4 & $1.41(1.34,1.48)^{* * *}$ & $0.95(0.89,1.00)$ & $1.05(0.92,1.21)$ \\
\hline \multicolumn{7}{|l|}{ Alcohol-related illness } \\
\hline No & 6367 & 708,326 & 8.99 & 1.00 & 1.00 & 1.00 \\
\hline Yes & 467 & 20,575 & 22.7 & $2.61(2.37,2.86)^{* * *}$ & $2.18(1.98,2.40)^{* * *}$ & $1.95(1.53,2.49)^{* * *}$ \\
\hline \multicolumn{7}{|l|}{ Anxiety } \\
\hline No & 3970 & 463,834 & 8.56 & 1.00 & 1.00 & 1.00 \\
\hline Yes & 2864 & 265,067 & 10.8 & $1.27(1.21,1.33)^{* * *}$ & $0.81(0.76,0.87)^{* * *}$ & $0.79(0.67,0.92)^{* *}$ \\
\hline
\end{tabular}

Rate per 1000 person-years

${ }^{a}:$ multivariable analysis of age, sex, and comorbidities of depression, mental disorders, insomnia, alcohol-related illness, and anxiety

${ }^{*} P<0.05,{ }^{* *} P<0.01,{ }^{* *} P<0.001$

medical visits annually in the patients with migraine. We found that only age was a mild positive predictor (0.04 higher frequency of medical visits) in patients with migraine (Table 5).

\section{Discussion}

In this study, Taiwanese patients with migraine demonstrated a lower all-cause subsequent mortality rate. Overall, 9.74 and 7.95 mortality events per 1000 person-years occurred in the comparison and total migraine cohorts. In the patients with NM, the protective effects against mortality might have been due to the frequent medical care and physical examinations, particularly for the female patients. Women usually tend to use more medical services and spend more on health care than men do, with regard to primary care, physical therapy, and other medical support $[15,16]$. However, the life expectancy for women generally being higher than that for men and the higher mortality risk among men can be confounding factors [17]. In the SM cohort, fewer protective effects were observed and those were noted only in patients aged $<50$ years; even the frequency of medical visits increased with age in these patients.

We observed some common trends between the increase of average all-cause hospitalization duration annually and higher mortality risk in the cohorts. In addition to the risk factors of age and the condition of $\mathrm{SM}$, alcohol-related illness, male sex, depression, and mental disorders were major risk factors for all-cause hospitalization and mortality in patients with migraine. 
Table 3 Incidence and hazard ratios of mortality stratified by age, sex, and comorbidity for patients with migraine comparing with those without migraine

\begin{tabular}{|c|c|c|c|c|c|c|c|c|c|c|c|}
\hline & \multirow{2}{*}{\multicolumn{2}{|c|}{$\begin{array}{l}\text { Comparison } \\
N=79,246\end{array}$}} & \multicolumn{9}{|l|}{ Migraine } \\
\hline & & & \multicolumn{3}{|l|}{$\begin{array}{l}\text { Total } \\
N=19,817\end{array}$} & \multicolumn{3}{|c|}{$\begin{array}{l}\text { Status migrainosus } \\
N=5868\end{array}$} & \multicolumn{3}{|c|}{$\begin{array}{l}\text { Non-status migraine } \\
N=13,949\end{array}$} \\
\hline & Event no & Rate & Event no & Rate & $\mathrm{HR}^{\mathrm{a}}(95 \% \mathrm{Cl})$ & Event no & Rate & $\mathrm{HR}^{\mathrm{a}}(95 \% \mathrm{Cl})$ & Event no & Rate & $\mathrm{HR}^{\mathrm{a}}(95 \% \mathrm{Cl})$ \\
\hline \multicolumn{12}{|l|}{ Age, year } \\
\hline $20-49$ & 1495 & 3.94 & 258 & 2.70 & $0.68(0.60,0.78)^{* * *}$ & 91 & 3.13 & $0.79(0.64,0.98)^{*}$ & 167 & 2.51 & $0.64(0.54,0.75)^{* * * *}$ \\
\hline $50-64$ & 1373 & 10.0 & 294 & 8.54 & $0.85(0.75,0.96)^{* *}$ & 86 & 8.53 & $0.85(0.68,1.06)$ & 208 & 8.55 & $0.85(0.73,0.98)^{*}$ \\
\hline $65+$ & 2799 & 42.6 & 615 & 36.7 & $0.86(0.79,0.94)^{* * *}$ & 224 & 42.0 & $0.97(0.85,1.11)$ & 391 & 34.2 & $0.81(0.73,0.90)^{* * *}$ \\
\hline \multicolumn{12}{|l|}{ Sex } \\
\hline Female & 3090 & 7.19 & 603 & 5.57 & $0.78(0.71,0.85)^{* * *}$ & 205 & 6.39 & $0.88(0.77,1.02)$ & 398 & 5.22 & $0.73(0.66,0.81)^{* * * *}$ \\
\hline Male & 2577 & 16.9 & 564 & 14.7 & $0.86(0.78,0.94)^{* *}$ & 196 & 15.8 & $0.89(0.77,1.03)$ & 368 & 14.1 & $0.84(0.75,0.94)^{* *}$ \\
\hline \multicolumn{12}{|l|}{ Comorbidity } \\
\hline None & 986 & 7.56 & 172 & 5.20 & $0.69(0.59,0.81)^{* * *}$ & 68 & 5.52 & $0.73(0.57,0.94)^{*}$ & 104 & 5.02 & $0.66(0.54,0.81)^{* * * *}$ \\
\hline With any one & 4681 & 10.4 & 995 & 8.75 & $0.84(0.79,0.90)^{* * *}$ & 333 & 10.4 & $1.00(0.89,1.11)$ & 662 & 8.12 & $0.78(0.72,0.85)^{* * *}$ \\
\hline
\end{tabular}

Rate per 1000 person-years

${ }^{a}$ : multivariable analysis including age, sex, and comorbidities of depression, mental disorders, insomnia, alcohol-related illness, and anxiety

${ }^{*} P<0.05,{ }^{* *} P<0.01,{ }^{* * *} P<0.001$

Among the comorbidities, alcohol-related illness was the strongest risk factor for hospital admission and mortality in patients with migraine. In general, a small amount of alcohol may not trigger migraine. Several recent studies could not conclusively show whether alcohol triggers migraines [18-20]. Differences in cultural and ethnic values and practices affect alcohol consumption, which increases complexity in studying the relationship between alcohol drinking and migraine. In this study, we considered alcohol-related illness a major comorbidity and risk factor; it should be due to that the patients with this comorbidity had already experienced toxic effects from alcohol consumption along with their migraine. Migraine alters peoples' neuroendocrinal, neurophysiological, and neuropsychological conditions [5-8], and increases the risk of cardiovascular diseases. In addition to cardiovascular risk, this study reveals that alcohol-related illness is another risk factor to cause premature mortality in Taiwanese patients with migraine.

Table 4 Stepwise regression analysis for average hospital days per year (all-cause admission) among patients with migraine

\begin{tabular}{llll}
\hline Variable & $\begin{array}{l}\text { Parameter } \\
\text { Estimate }\end{array}$ & $\begin{array}{l}\text { Standard } \\
\text { Error }\end{array}$ & $95 \% \mathrm{Cl}$ \\
\hline Intercept & -3.71 & 0.30 & $(-4.30,-3.12)^{* * *}$ \\
Status migrainosus & 0.66 & 0.20 & $(0.27,1.05)^{* * *}$ \\
Age & 0.11 & 0.01 & $(0.09,0.12)^{* * *}$ \\
Sex (male vs. female) & 1.98 & 0.21 & $(1.57,2.39)^{* * *}$ \\
Alcohol-related illness & 4.10 & 0.49 & $(3.15,5.06)^{* * *}$ \\
Mental disorders & 0.81 & 0.20 & $(0.41,1.21)^{* * *}$ \\
Depression & 1.08 & 0.29 & $(0.51,1.65)^{* * *}$ \\
\hline
\end{tabular}

***P $<0.001$
To our knowledge, this is the first study to show the long-term mortality rates in patients with migraine: patients with SM and NM demonstrated diverse survival outcomes. After the NHI was implemented in Taiwan, the annual mortality rate in general population significantly decreased by $5.83 \%$ from 1996 to 1999 [21]. In this study, we only analyzed the mortality risks in patients with migraine who died after being treated at inpatient facilities, excluding mortality cases outside a hospital. The NHI program has been over 20 years which is a universally compulsive insurance covering more than $99 \%$ of Taiwan's population and it is operated by the government in Taiwan. NHI guarantees the residents of Taiwan equal access of medical service regardless of socioeconomic status, background, and critical problems existing or not $[12,22]$. The Taiwan's universal healthcare system showed very few disparities in accessing inpatient service and ultimate outcomes between different hospitals and areas in Taiwan [23, 24], and the proportion of migraineurs died outside a hospital should be very rare. However, the detail underlying mechanisms need to be studied in the future to explain why Taiwanese patients with migraine have a lower mortality risk depicted in this study. Furthermore, patients ever with SM, of the male sex, and with alcohol-related illness, depression, or mental disorders have fewer protective

Table 5 Stepwise regression analysis for frequency of migrainerelated visits per year among patients with migraine

\begin{tabular}{llll}
\hline Variable & Parameter Estimate & Standard Error & $95 \% \mathrm{Cl}$ \\
\hline Intercept & -0.49 & 0.91 & $(-2.29,1.30)$ \\
Age & 0.04 & 0.02 & $(0.002,0.08)^{*}$ \\
${ }^{* P}<0.05$ & & &
\end{tabular}


effects from premature mortality, and could be said to have a malignant course of migraine. We hope that our findings highlight a future strategy for providing proper medical care to patients with migraine.

Because of our study design, which included a nationwide, population-based sample with little risk of recall and selection bias, our findings should be considered convincing in Taiwan and other developing Asian countries with a heritage similar to that of Taiwan. However, this study has several limitations. First, we could not directly contact our patients because their identities were anonymized in the LHID. Therefore, the study design did not include details regarding the migraines, such as duration and frequency, psychological burden, and whether or how the migraines were treated with medication. Whether any migraine medications and other comorbid disorders influence the mortality rate is unknown. Second, any mortality cases occurring outside a hospital were out of the scope of our study. We intentionally designed our study in those died after being treated at inpatient facilities to achieve results with high validity, despite the slight possibility of introducing underestimation bias into the results. Third, we have to admit there was a possible bias that missed or underdiagnosed SM and NM. However, the NHIRD covers a highly representative sample of Taiwan's general population because the reimbursement policy is universal and operated by a single-buyer, the government in Taiwan. All insurance claims should be scrutinized by medical reimbursement specialists and peer review according to the standard diagnosed criteria in the study. If these doctors or hospitals make wrong diagnoses or coding, they will be punished with a lot of penalties. Therefore, the diagnoses of SM and NM in this study were highly reliable and our results indicate that the sample size was sufficient to statistically demonstrate the mortality risk in patients with migraine in Taiwan.

\section{Conclusions}

This study revealed that Taiwanese patients with migraine benefit from comprehensive and universal health care for not only controlling their migraines but also reducing subsequent mortality risk. Our findings provide vital information for clinicians and for assisting global efforts for understanding and treating migraine.

\section{Abbreviations}

aHR: Adjusted hazard ratio; Cl: Confidence interval; ICD-9-CM: International Classification of Diseases, Ninth Revision, Clinical Modification;

NHIRD: National Health Insurance Research Database

\section{Acknowledgements}

This work was supported by grants from the Ministry of Health and Welfare, Taiwan (MOHW107-TDU-B-212-123004), China Medical University Hospital (DMR-107-192); Academia Sinica Stroke Biosignature Project (BM10701010021); MOST Clinical Trial Consortium for Stroke (MOST 106-2321-B-039-005-); Tseng-Lien Lin Foundation, Taichung, Taiwan; and Katsuzo and Kiyo Aoshima Memorial
Funds, Japan. The funders had no role in study design, data collection and analysis, decision to publish, or preparation of the manuscript. No additional external funding received for this study.

\section{Availability of data and materials}

The dataset used in this study is held by the Taiwan Ministry of Health and Welfare (MOHW). The Ministry of Health and Welfare must approve our application to access this data. Any researcher interested in accessing this dataset can submit an application form to the Ministry of Health and Welfare requesting access. Please contact the staff of MOHW (Email: stcarolwu@mohw.gov.tw) for further assistance. Taiwan Ministry of Health and Welfare Address: No.488, Sec. 6, Zhongxiao E. Rd. Nangang Dist., Taipei City 115, Taiwan (R.O.C.). Phone: + 886-2-8590-6848. All relevant data are within the paper.

\section{Authors' contributions}

All authors have contributed significantly, and that all authors are in agreement with the content of the manuscript: Conception/Design: TH, C-HK; Provision of study materials: C-HK; Collection and/or assembly of data: All authors; Data analysis and interpretation: All authors; Manuscript writing: All authors; Final approval of manuscript: All authors.

\section{Ethics approval and consent to participate}

The NHIRD encrypts patient personal information to protect privacy and provides researchers with anonymous identification numbers associated with relevant claims information, including sex, date of birth, medical services received, and prescriptions. Therefore, patient consent is not required to access the NHIRD. This study was approved to fulfill the condition for exemption by the Institutional Review Board (IRB) of China Medical University (CMUH104-REC2-115-CR2). The IRB also specifically waived the consent requirement.

Consent for publication

Not available.

\section{Competing interests}

The authors declare that they have no competing interests.

\section{Publisher's Note}

Springer Nature remains neutral with regard to jurisdictional claims in published maps and institutional affiliations.

\section{Author details}

${ }^{1}$ Department of Neurosurgery, Hualien Tzu Chi General Hospital, Buddhist Tzu Chi Medical Foundation, Hualien, Taiwan. ${ }^{2}$ College of Medicine, Tzu Chi University, Hualien, Taiwan. ${ }^{3}$ Management Office for Health Data, China Medical University Hospital, Taichung, Taiwan. ${ }^{4}$ College of Medicine, China Medical University, Taichung, Taiwan. ${ }^{5}$ Graduate Institute of Biomedical Sciences and School of Medicine, College of Medicine, China Medical University, No. 2, Yuh-Der Road, Taichung 404, Taiwan. ${ }^{6}$ Department of Nuclear Medicine and PET Center, China Medical University Hospital, Taichung, Taiwan. ${ }^{7}$ Department of Bioinformatics and Medical Engineering, Asia University, Taichung, Taiwan.

Received: 21 June 2018 Accepted: 13 July 2018

Published online: 25 July 2018

\section{References}

1. Streel S, Donneau AF, Hoge A, Albert A, Schoenen J, Guillaume M (2015) One-year prevalence of migraine using a validated extended French version of the ID migraine ${ }^{\mathrm{TM}}$ : a Belgian population-based study. Rev Neurol (Paris) 171:707-714

2. Stovner LJ, Zwart JA, Hagen K, Terwindt GM, Pascual J (2006) Epidemiology of headache in Europe. Eur J Neurol 13:333-345

3. Wang SJ, Chung CS, Chankrachang S, Ravishankar K, Merican JS, Salazar G, Siow C, Cheung RT, Phanthumchinda K, Sakai F (2008) Migraine disability awareness campaign in Asia: migraine assessment for prophylaxis. Headache 48:1356-1365

4. Wang SJ, Fuh JL, Young YH, Lu SR, Shia BC (2000) Prevalence of migraine in Taipei, Taiwan: a population-based survey. Cephalalgia 20:566-572

5. Lipton RB, Stewart WF, Diamond S, Diamond ML, Reed M (2001) Prevalence and burden of migraine in the United States: data from the American migraine study II. Headache 41:646-657 
6. Rambarat CA, Elgendy IY, Johnson BD, Reis SE, Thompson DV, Sharaf BL, Bittner V, Sopko G, Bairey Merz CN, Pepine CJ, Ahmed B (2017) Migraine headache and long-term cardiovascular outcomes: an extended follow-up of the women's ischemia syndrome evaluation. Am J Med 130:738-743

7. Nović A, Kölves K, O'Dwyer S, De Leo D (2016) Migraine and suicidal behaviors: a systematic literature review. Clin J Pain 32:351-364

8. Leonardi M (2015) Burden of migraine: what should we say more? Neurol Sci 36(Suppl 1):1-3

9. Granella F, Cavallini A, Sandrini G, Manzoni GC, Nappi G (1998) Long-term outcome of migraine. Cephalalgia 18:30-33

10. Colombo B, Dalla Libera D, Dalla Costa G, Comi G (2013) Refractory migraine: the role of the physician in assessment and treatment of a problematic disease. Neurol Sci 34(Suppl 1):109-112

11. Database NHIR. Taiwan. http://nhird.nhri.org.tw/en/index.html (cited in 2017)

12. Ministry of Health and Welfare (2017). Taiwan health and welfare report. available at: http://www.mohw.gov.tw

13. Lin FY, Yang YC, Lin CL, Lee LJ (2018) Increased risk of overactive bladder in patients with idiopathic Parkinson's disease: insight from a nationwide population-based cohort study. PLoS One 13:e0193783. https://doi.org/10. 1371/journal.pone.0193783 eCollection 2018

14. Lin SY, Lin CL, Lin CC, Wang IK, Hsu WH, Kao CH (2018) Risk of acute coronary syndrome and peripheral arterial disease in chronic liver disease and cirrhosis: a nationwide population-based study. Atherosclerosis 270: 154-159. https://doi.org/10.1016/j.atherosclerosis.2018.01.047

15. Kaur S, Stechuchak KM, Coffman CJ, Allen KD, Bastian LA (2007) Gender differences in health care utilization among veterans with chronic pain. J Gen Intern Med 22:228-233

16. Owens GM (2008) Gender differences in health care expenditures, resource utilization, and quality of care. J Manag Care Pharm 14(Supp I):2-6

17. Kochanek KD, Murphy SL, Xu J, Arias E (2015) Mortality in the United States, 2014. NCHS Data Brief 229:1-8

18. Dueland AN (2015) Headache and alcohol. Headache 55:1045-1049

19. Panconesi A, Franchini M, Bartolozzi ML, Mugnai S, Guidi L (2013) Alcoholic drinks as triggers in primary headaches. Pain Med 14:1254-1259

20. Krymchantowski AV, da Cunha Jevoux C (2014) Wine and headache. Headache 54:967-975

21. Lee YC, Huang YT, Tsai YW, Huang SM, Kuo KN, McKee M, Nolte E (2010) The impact of universal National Health Insurance on population health: the experience of Taiwan. BMC Health Serv Res 10:225

22. Lee YH, Ang TFA, Chiang TC, Kaplan WA (2018) Growing concerns and controversies to Taiwan National Health Insurance- what are the lessons from mainland China, South Korea and Singapore? Int J Health Plann Manag 33:e357-e366. https://doi.org/10.1002/hpm.2387

23. Huang N, Yip W, Chang HJ, Chou YJ (2006) Trends in rural and urban differentials in incidence rates for ruptured appendicitis under the National Health Insurance in Taiwan. Public Health 120:1055-1063

24. Shu CC, Lin JW, Lin YF, Hsu NC, Ko WJ (2011) Evaluating the performance of a hospitalist system in Taiwan: a pioneer study for nationwide health insurance in Asia. J Hosp Med 6:378-382

Ready to submit your research? Choose BMC and benefit from:

- fast, convenient online submission

- thorough peer review by experienced researchers in your field

- rapid publication on acceptance

- support for research data, including large and complex data types

- gold Open Access which fosters wider collaboration and increased citations

- maximum visibility for your research: over $100 \mathrm{M}$ website views per year

At $\mathrm{BMC}$, research is always in progress.

Learn more biomedcentral.com/submissions 